GANIT J. Bangladesh Math. Soc. (ISSN 1606-3694) 37 (2017) 29-37

\title{
A STUDY ON TIDAL POTENTIAL AND TIDE GENERATING FORCE
}

\author{
M. Mizanur Rahman ${ }^{1, *}$, Gour Chandra Paul ${ }^{2}$ and Ashabul Hoque ${ }^{3}$ \\ ${ }^{1}$ Department of Mathematics, Shahjalal University of Science \& Technology, \\ Sylhet-3114, Bangladesh \\ ${ }^{2,3}$ Department of Mathematics, University of Rajshahi, Rajshahi 6205, Bangladesh \\ *Corresponding author: mizanmath_sust@yahoapo.com
}

Received 07.05.2016 Accepted 25.05.2017

\begin{abstract}
This study deals with the derivation of tidal potential and tide generating forces. Tidal potential is derived from the gravitational attraction of masses of the moon and the sun and from this potential tide generating forces are derived taking its horizontal gradients.
\end{abstract}

Keywords: Gravitational attraction; Low water; High water; Tidal potential; Tide generating forces.

\section{Introduction}

The phenomenon of oceanic tides has been observed and studied by humanity ever since the dawn of civilization. Obviously people must early have noticed the connection between high and low water and the position of the moon and the sun. Due to regularity of phenomena it became closely associated with the flow of time as the very name tides indicates. The established equilibrium theory due to Newton [7] explains well the forces that generate the tides. Newton's equilibrium theory of tides also explains the observed dominant semidiurnal periodicity of ocean tides. Up to then it had been a mystery that high water occurs both with the moon overhead and also about 12 hours later when the moon is on the other side of the earth. Today Newton's equilibrium theory provides the correct tide generating force to which the oceans respond hydro dynamically in a rather complicated fashion. Although Newton discovered the true astronomic nature of tide, it was Laplace [5] who derived the first hydrodynamic equations of ocean tides. Laplace's tidal equations contain the tide generating force in terms of Newton's equilibrium tide as the forcing function. The reviews by Cartwright [1], Schwiderski [9, 10] and Davies et al. [2] survey central parts of the literature. Again if the earth were an ocean planet with no land, and of the ocean were very deep, the two process would produce a pair of bulges of water on earth, one on the side facing the moon, one on side away the moon. Considering these facts, in this study a clear derivation of the forces is given and the derivation of tidal potential and tide generating forces are presented in detail.

\section{Tide Generating Force and Tidal Potential}

Tidal oscillations are the response of the ocean and the earth to the gravitational pull of the other celestial bodies. These oscillations can be calculated from hydrodynamic equations for a self- 
gravitating ocean on the rotating and elastic earth. The field derive tides is called the tidal potential and it rises mainly from the gravitational attraction of the moon and the sun in which other heavenly bodies other than the earth also take part. If the earth were an ocean planet without land and if the influence of inertia and currents are ignored, the gravity gradient produces a pair of bulges of water on earth, one on the side facing the moon or sun, one on the side away from the moon or sun. Here, following the discussion in Pugh [8] and Stewart [11], a clear derivation of the forces is given.

\section{Derivation of tide generating potential}

Firstly, tides generated by the gravitational attraction of the moon are calculated. If the effects due to the diurnal rotation of the earth are ignored, the potential at a point $\mathrm{P}$ on the surface of the earth, shown in Fig.1, due to the gravitational pull of the moon is given by (see Nakamura et al. [6])

$$
V_{M}=-\frac{G M}{r_{1}}
$$

where $\mathrm{M}$ is the mass of the moon, $\mathrm{G}$ is the universal gravitational constant, $\mathrm{R}$ is the distance of the earth centre from that of the moon, and $r_{1}$ is the distance from the centre of the moon to the point $\mathrm{P}$.

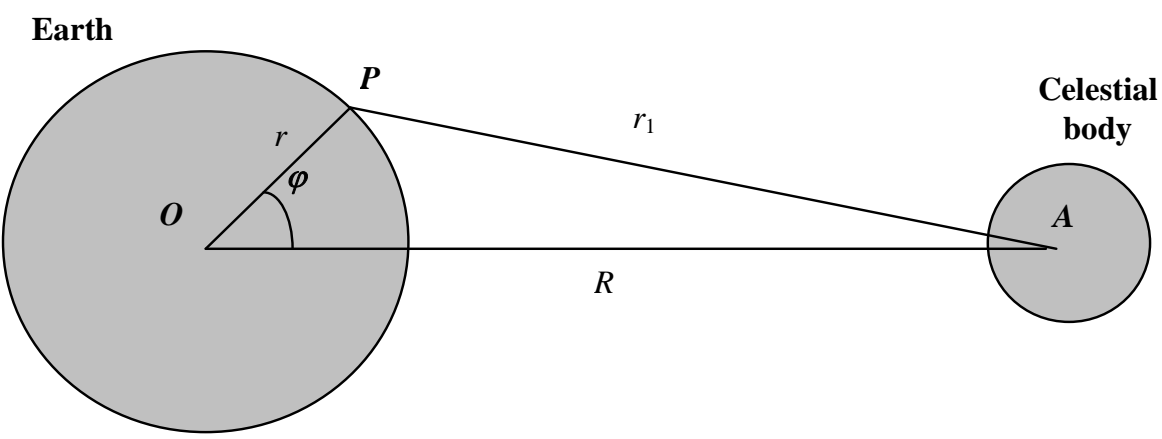

Figure 1: Diagram to show the location of the general point $P$ on the earth's surface.

Using the cosine formula in triangle OPA (Fig.1), we get

$$
r_{1}^{2}=r^{2}+R^{2}-2 r R \cos \varphi
$$

Using this relation in Eq. (1), we have

$$
V_{M}=-\frac{G M}{R}\left(1-2 \frac{r}{R} \cos \varphi+\frac{r^{2}}{R^{2}}\right)^{-\frac{1}{2}}
$$

Here,

$$
\left(1-2 \frac{r}{R} \cos \varphi+\frac{r^{2}}{R^{2}}\right)^{-\frac{1}{2}}
$$


$=1+\frac{r}{R} \cos \varphi-\frac{r^{2}}{2 R^{2}}+\frac{3}{4.2 !}\left(\frac{4 r^{2}}{R^{2}} \cos ^{2} \varphi-4 \frac{r^{3}}{R^{3}} \cos \varphi+\ldots\right)+\frac{15}{8.3 !}\left(\left(2 \frac{r}{R} \cos \varphi\right)^{3}-\ldots\right)+\ldots$
or, $\left(1-2 \frac{r}{R} \cos \varphi+\frac{r^{2}}{R^{2}}\right)^{-\frac{1}{2}}=1+\frac{r}{R} \cos \varphi+\frac{r^{2}}{R^{2}}\left\{\frac{1}{2}\left(3 \cos ^{2} \varphi-1\right)\right\}+\frac{r^{3}}{R^{3}}\left\{\frac{1}{2}\left(5 \cos ^{3} \varphi-3 \cos \varphi\right)\right\}+\ldots$

Thus the tidal potential due to the gravitational pull of the moon given by Eq. (2) can be written as

$V_{M}=-\frac{G M}{R}\left\{1+V_{0}(\cos \varphi)+\frac{r^{2}}{R^{2}} V_{1}(\cos \varphi)+\frac{r^{3}}{R^{3}} V_{2}(\cos \varphi)\right\}+\ldots$,

where $V_{0}=\cos \varphi, V_{1}=\frac{1}{2}\left(3 \cos ^{2} \varphi-1\right), V_{2}=\frac{1}{2}\left(5 \cos ^{3} \varphi-3 \cos \varphi\right)$.

The first term in Eq. (3) is constant and so has no associated force. The second term generates a uniform force parallel to OA. This can easily be confirmed by differentiating Eq. (3) with regard to $R \cos \varphi$, which gives

$$
-\frac{\partial V_{M}}{\partial(\cos \varphi)}=-\frac{G M}{R^{2}}
$$

The third term of Eq. (3) is the major tide generating term. The fourth and higher terms may be neglected because $\frac{r}{R} \approx \frac{1}{60}$. Therefore the effective tide generating potential due to the gravitational pull of the moon can be given by

$$
V_{M}=-\frac{G M r^{2}}{2 R^{3}}\left(3 \cos ^{2} \varphi-1\right) .
$$

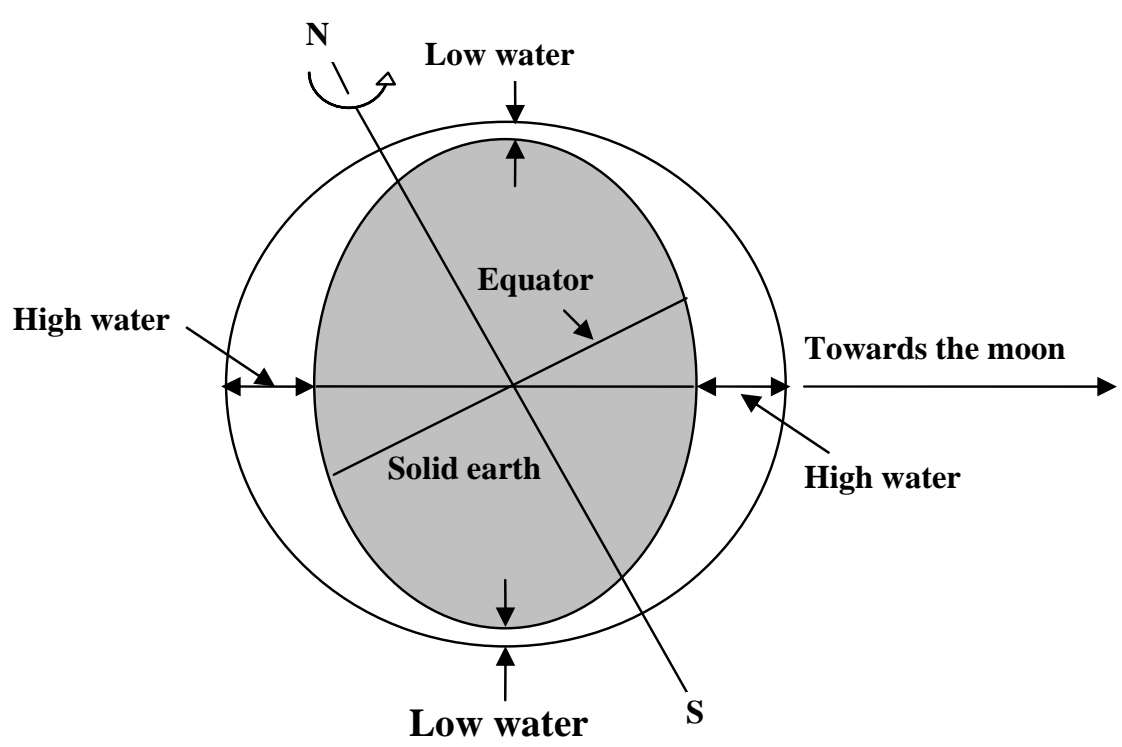

Figure 2: Rotation of the earth with respect to its axis. 
But the earth is not fixed; it spins (Fig. 2). Therefore, the changing of tidal potential at a fixed geographic coordinate on the earth is needed to be determined. This can easily be determined from the astronomical establishment (see Fig. 3)

$$
\cos \varphi=\sin \phi \sin \delta+\cos \phi \cos \delta \cos \tau
$$

In Eq. (5), $\phi$ is the latitude or lunar angle, $\delta$ is the declination of the moon north of the equator whose effects are shown in Fig. 4, and $\tau$ is an hour angle of the moon.

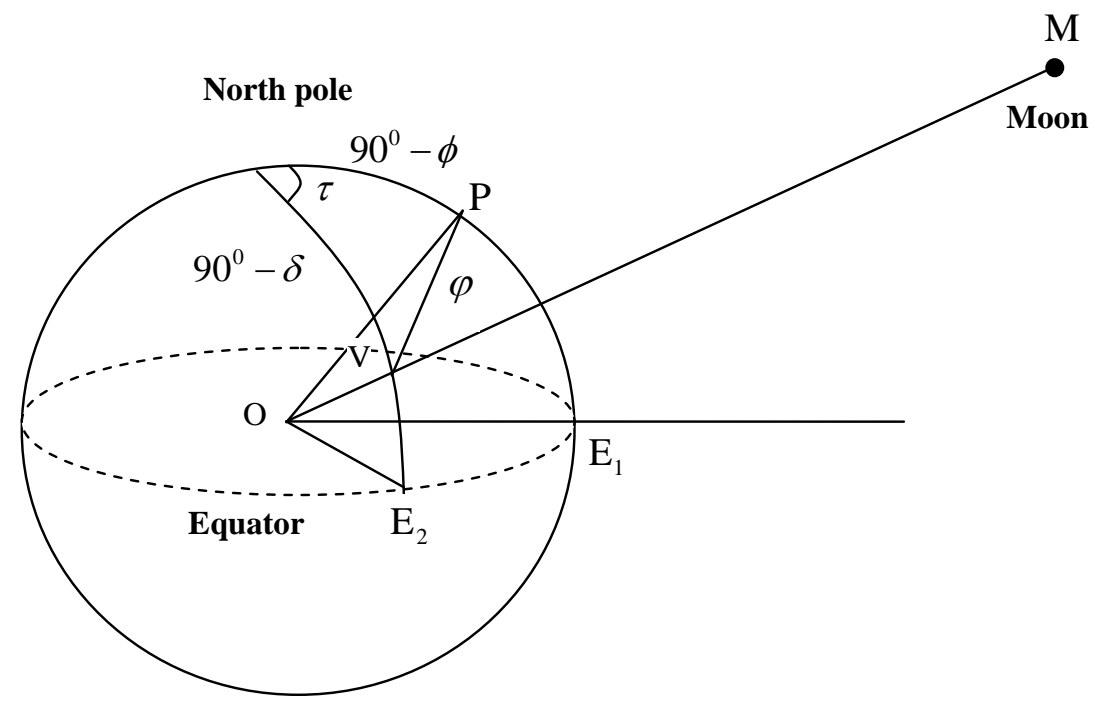

Figure 3 Location of P relative to the sub-lunar point V.

The declination and Greenwich hour angle (GHA) are the coordinates, shown graphically in Fig. 5, (latitude and longitude) of the geographical position of the sun or the moon, where the line from the sun or moon to the centre of the earth intersects its surface. The local hour angle is: LHA = GHA +/- longitude.

Substituting for $\cos \varphi$ in Eq. (4), we have

$$
\begin{aligned}
& V_{M}=-\frac{G M r^{2}}{2 R^{3}}\left(3 \cos ^{2} \varphi-1\right) . \\
& =-\frac{3 G M r^{2}}{2 R^{3}}\left(\cos ^{2} \varphi-\frac{1}{3}\right) \\
& =-\frac{3 G M r^{2}}{2 R^{3}}\left((\sin \phi \sin \delta+\cos \phi \cos \delta \cos \tau)^{2}-\frac{1}{3}\right) \\
& =-\frac{3 G M r^{2}}{2 R^{3}}\left(\left(\sin ^{2} \phi \sin ^{2} \delta+\frac{1}{2} \cos ^{2} \phi+\cos ^{2} \delta+\frac{1}{2} \cos ^{2} \phi \cos ^{2} \delta \cos 2 \tau+\frac{1}{2} \sin 2 \phi \sin 2 \delta \cos \tau\right)-\frac{1}{3}\right)
\end{aligned}
$$




$$
\begin{aligned}
& =-\frac{3 G M r^{2}}{4 R^{3}}\left(\left(2 \sin ^{2} \phi \sin ^{2} \delta+\cos ^{2} \phi \cos ^{2} \delta+\cos ^{2} \phi \cos ^{2} \delta \cos 2 \tau+\sin 2 \phi \sin 2 \delta \cos \tau\right)-\frac{2}{3}\right) \\
& =-\frac{3 G M r^{2}}{4 R^{3}}\left(\left(2 \sin ^{2} \phi \sin ^{2} \delta+\left(1-\sin ^{2} \phi\right)\left(1-\sin ^{2} \delta\right)+\cos ^{2} \phi \cos ^{2} \delta \cos 2 \tau+\sin 2 \phi \sin 2 \delta \cos \tau\right)-\frac{2}{3}\right) \\
& =-\frac{3 G M r^{2}}{4 R^{3}}\left(\left(3 \sin ^{2} \phi \sin ^{2} \delta+1-\sin ^{2} \phi-\sin ^{2} \delta+\cos ^{2} \phi \cos ^{2} \delta \cos 2 \tau+\sin 2 \phi \sin 2 \delta \cos \tau\right)-\frac{2}{3}\right) \\
& =-\frac{3 G M r^{2}}{4 R^{3}}\left[\frac{9 \sin ^{2} \phi \sin ^{2} \delta-3 \sin ^{2} \phi-3 \sin ^{2} \delta+1}{3}+\cos ^{2} \phi \cos ^{2} \delta \cos 2 \tau+\sin 2 \phi \sin 2 \delta \cos \tau\right] \\
& =-\frac{3 G M r^{2}}{4 R^{3}}\left[\frac{\left(3 \sin ^{2} \phi-1\right)\left(3 \sin ^{2} \delta-1\right)}{3}+\cos ^{2} \phi \cos ^{2} \delta \cos 2 \tau+\sin 2 \phi \sin 2 \delta \cos \tau\right] \\
& =-\frac{G M r^{2}}{4 R^{3}}\left[\frac{\left(3 \sin ^{2} \phi\right)\left(3 \sin ^{2} \delta-1\right)}{3}+\cos ^{2} \phi \cos ^{2} \delta \cos ^{2} \tau+\sin ^{2} \phi \sin ^{2} \delta \cos \tau\right]
\end{aligned}
$$

Similar results can be determined due to the gravitational pull exerted by the sun. Therefore, if we use subscripts $M$ and $S$ for the moon and the sun respectively, the tidal potentials due to the attractions of the moon and the sun are respectively written as

$$
\begin{aligned}
& V_{M}=G_{M}^{\prime}\left[\frac{\left(3 \sin ^{2} \phi-1\right)\left(3 \sin ^{2} \delta_{M}-1\right)}{3}+\cos ^{2} \phi \cos ^{2} \delta_{M} \cos 2 \tau_{M}+\sin 2 \phi \sin 2 \delta_{M} \cos \tau_{M}\right], \\
& V_{M}=G_{M}^{\prime}\left[\frac{\left(3 \sin ^{2} \phi-1\right)\left(3 \sin ^{2} \delta_{S}-1\right)}{3}+\cos ^{2} \phi \cos ^{2} \delta_{S} \cos ^{2} \tau_{S}+\sin ^{2} \phi \sin ^{2} \delta_{S} \cos \tau_{S}\right]
\end{aligned}
$$

where $G_{M}^{\prime}=-\frac{G M_{M} r_{M}^{2}}{4 R_{M}^{3}}$ and $G_{S}^{\prime}=-\frac{G M_{S} r_{S}^{2}}{4 R_{S}^{3}}$.

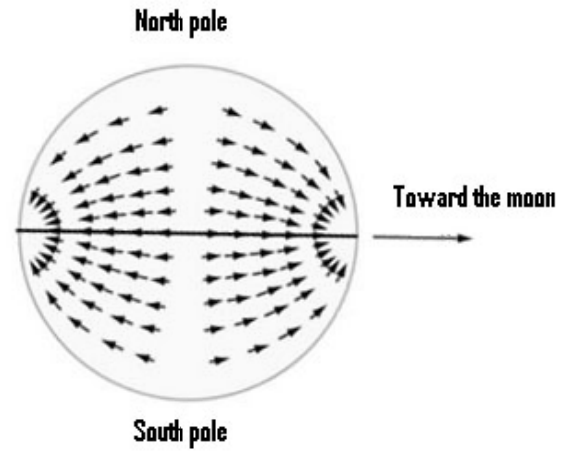

(a)

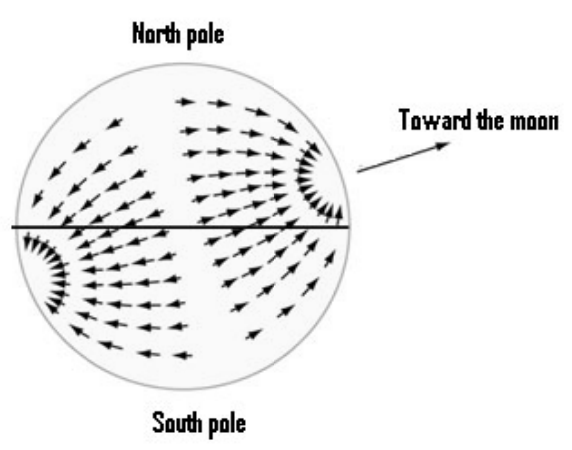

(b) 


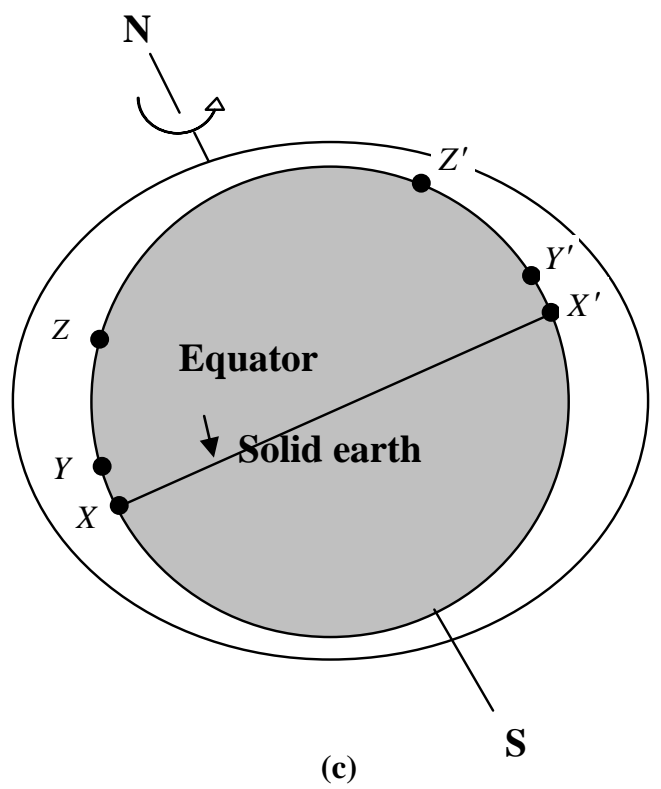

Figure 4 Effects of the declination of the moon.
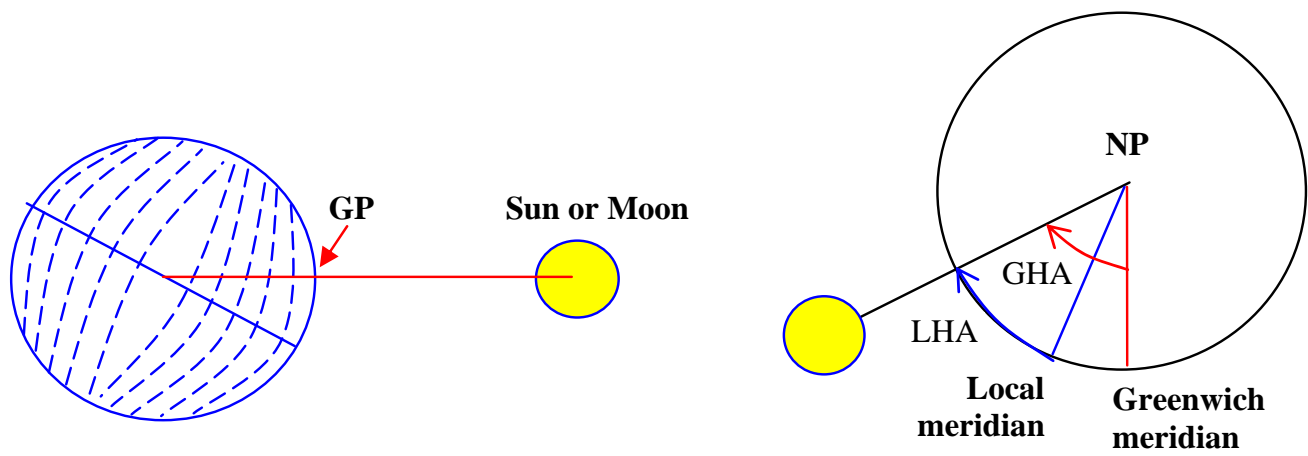

Figure 5 Declination of the sun or moon and Greenwich hour angle.

Then, the total tidal potential of the moon and the sun for the earth is thus given by

$$
\begin{aligned}
V= & V_{N}+V_{M} \\
= & -\frac{\left(3 \sin ^{2} \phi-1\right)\left[3\left(G_{M}^{\prime} \sin ^{2} \delta_{M}+G_{S}^{\prime} \sin ^{2} \delta_{S}\right)-\left(G_{M}^{\prime}+G_{S}^{\prime}\right)\right]}{3} \\
& -\cos ^{2} \phi\left(G_{M}^{\prime} \cos ^{2} \delta_{M} \cos ^{2} \tau_{M}+G_{S}^{\prime} \cos ^{2} \delta_{S} \cos ^{2} \tau_{S}\right) \\
& -\sin ^{2} \phi\left(G_{M}^{\prime} \sin ^{2} \delta_{M} \cos ^{2} \tau_{M}+G_{S}^{\prime} \sin ^{2} \delta_{S} \cos ^{2} \tau_{S}\right)
\end{aligned}
$$


In Eq. (6), the terms related to $\cos ^{2} \phi$ is termed as semi-diurnal tides, while the terms consist of $\sin ^{2} \phi$ are called the diurnal tides and the terms related to $3 \sin ^{2} \phi-1$ are long-period tides. For the lunar tidal potential, those periods are approximately 12 hours, 24 hours and 14 days respectively. For the solar tidal potential, they are close to 12 hours, 24 hours and 180 days respectively.

Doodson [3] expanded Eq. (6) into Fourier series by choosing frequencies shown in Table 1 (Stewart [11]):

Table 1 Fundamental tidal frequencies.

\begin{tabular}{|l|l|l|l|}
\hline & Frequency (degree/hour) & Period & Source and mening \\
\hline$f_{1}$ & 14.49205211 & 1 lunar day & Local mean lunar time \\
\hline$f_{2}$ & 0.54901653 & 1 month & Mean longitude of the Moon \\
\hline$f_{3}$ & 0.04106864 & 1 year & Mean longitude of the Sun \\
\hline$f_{4}$ & 0.00464184 & 8.847 years & Longitude of the Moon's perigee \\
\hline$f_{5}$ & -0.00220641 & 10.613 years & Longitude of the Moon's ascending note \\
\hline$f_{6}$ & 0.000000196 & 20.940 years & Longitude of the Sun's perigee \\
\hline
\end{tabular}

Table 2 Constants associated with major tidal constituents.

\begin{tabular}{|c|c|c|c|c|c|c|c|c|c|}
\hline $\begin{array}{l}\text { Tidal } \\
\text { species }\end{array}$ & $\begin{array}{l}\text { Darwin } \\
\text { name }\end{array}$ & $n_{1}$ & $n_{2}$ & $n_{3}$ & $n_{4}$ & $n_{5}$ & $n_{6}$ & $\begin{array}{l}\text { Equilibrium } \\
\text { Amplitude }\end{array}$ & period \\
\hline Semi-diurnal & $n_{1}=2$ & & & & & & & & \\
\hline Principal lunar & $M_{2}$ & 2 & 0 & 0 & 0 & 0 & 0 & 0.242334 & 12.4206 \\
\hline Principal solar & $S_{2}$ & 2 & 2 & -2 & 0 & 0 & 0 & 0.112841 & 12.0000 \\
\hline Lunar elliptic & $N_{2}$ & 2 & -2 & 0 & 1 & 0 & 0 & 0.046398 & 12.6584 \\
\hline Lunisolar & $K_{2}$ & 2 & 2 & 0 & 0 & 0 & 0 & 0.030704 & 11.9673 \\
\hline Diurnal & $n_{1}=1$ & & & & & & & & \\
\hline Lunisolar & $K_{1}$ & 1 & 1 & 0 & 0 & 0 & 0 & 0.141565 & 23.9344 \\
\hline Principal lunar & $O_{1}$ & 1 & -1 & 0 & 0 & 0 & 0 & 0.100514 & 25.8194 \\
\hline Principal solar & $P_{1}$ & 1 & 1 & -2 & 0 & 0 & 0 & 0.016813 & 21.0659 \\
\hline Lunar elliptic & $Q_{1}$ & 1 & -2 & 0 & 1 & 0 & 0 & 0.019256 & 26.8681 \\
\hline Long period & $n_{1}=0$ & & & & & & & & \\
\hline Fortnightly & $M_{f}$ & 0 & 2 & 0 & 0 & 0 & 0 & 0.041742 & 327.85 \\
\hline Monthly & $M_{m}$ & 0 & 1 & 0 & -1 & 0 & 0 & 0.022026 & 661.31 \\
\hline Semi-annual & $S_{s a}$ & 0 & 0 & 2 & 0 & 0 & 0 & 0.019446 & 4383.05 \\
\hline
\end{tabular}

Doodson's expansion, however, leads to an elegant decomposition of tidal constituents into groups with similar frequencies and spatial variability. Each tidal constituent has a particular frequency with different Doodson integer numbers $n_{i}$ so that 


$$
f=n_{1} f_{1}+n_{2} f_{2}+n_{3} f_{3}+n_{4} f_{4}+n_{5} f_{5}+n_{6} f_{6} \text {. }
$$

If the ocean surface is equilibrium with tidal potential, the largest tidal constituents would have the equilibrium amplitude in the Table 2 . Individual tidal constituent has its equilibrium equation. The tide generating forces form the tidal potential are thus given by

$$
\left.\begin{array}{l}
F_{x}=\frac{\delta V}{\partial x}=-g \frac{\delta \tau}{\partial x} \\
F_{y}=\frac{\delta V}{\partial y}=-g \frac{\delta \tau}{\partial y}
\end{array}\right\} .
$$

As according to Pugh [8], $V=-g \tau(x, y)$.

According to Kowalik and Proshutinsky [4], for diurnal species

$$
\tau_{i}(x, y)=K_{i} \sin ^{2} \phi \cos \left(\omega_{i} t+\kappa_{i}+\lambda\right),
$$

for semi-diurnal species

$$
\tau_{i}(x, y)=K_{i} \cos ^{2} \phi \cos \left(\omega_{i} t+\kappa_{i}+2 \lambda\right),
$$

and for long period species

$$
\tau_{i}(x, y)=K_{i}\left(\frac{3}{2} \cos ^{2} \phi-1\right) \cos \left(\omega_{i} t+\kappa_{i}\right) .
$$

In the above Eqs. (9-11), $K_{i}, \omega_{i}$ and $\kappa_{i}$ are tidal amplitudes, frequencs and astronomical arguments respectively and $\phi$ and $\lambda$ are latitude and longitude of the interest position respectively. The values of the constants $K_{i}, \omega_{i}$ and $\kappa_{i}$ are shown in Table 2 (see, Kowalik and Proshutinsky [4]).

\section{Conclusion}

Sea level variation is of major concern in Bangladesh because vast low-lying area of the country experiences large tidal range, heavy river run-off and frequent severe storm surges. For the study of sea level rise accuracy of tidal data is an essential prerequisite. The present study will provide good information about the tidal potential and tide generating force along the coastal belt of Bangladesh. The findings of the study can be applied in storm surge modeling.

\section{REFERENCES}

[1] Cartwright, E.D., Ocenic tides. Rep. Prog. Phy. 40 (1977), 665-708.

[2] Davis M.A. Jones J.E. and Xing, J. A review of recent development in tidal hydrodynamic modelling, Part I and part II. J. Hydraul. Eng- ASCE 123 (1996), 278-302.

[3] Doodson A. T., The harmonic development of the tide-generating potential. Proc. Roy. Soc., London, Ser. A, 100 (1921), 305. 
[4] Kowalik Z. Proshutinsky T. and Proshutinsky, A. Tide-tsunami interactions. Science of Tsunami Hazards 24 (2006), 242-256.

[5] Laplace, P.S., Recherches sur quelques points de system du monde. Men. Acad. Roy. Sci. 88 (1775).

[6] Nakamura T. Awaji T. Hatayama T. Akitomo K. and Takizawa, T., Tidal Exchange through the Kuril Straits. J. Phys. Oceanogr. 30 (2000), 1622-1644.

[7] Newton, I., Philosophiae Naturalis Principia Mathematica. London, 1687.

[8] Pugh, D. T., Tides, Surges and Mean Sea Level. Natural Environment Research Council. Swindon, UK, 1987.

[9] Schwiderski, E.W., On Charting Global Ocean Tides. Rev. Geophys. And Space Physics 18 (1980), 243-268.

[10] Schwiderski, E.W., Tides. In The Nordic Seas: New York, Springer Verlag (1986), 191-209.

[11] Stewart, R.H., Introduction to Physical Oceanography. Texas A \& M University, 353p. 2007. 